\title{
Sistema de Informação para Classificação de Clientes
}

\author{
FREIRE, Luiz Claudio Mandarino ${ }^{1}$; DALTO, Edson José2 \\ ${ }^{1}$ Faculdades IBMEC - mandarin@ism.com.br \\ ${ }^{2}$ Dalto / Faculdades IBMEC - edalto@ibmecrj.br
}

\begin{abstract}
Resumo
O acirramento da competição dos mercados incentivou as empresas a investirem na criação de novos produtos e serviços como forma de diferenciação. Conseqüentemente, os custos de atendimento aumentaram, gerando a necessidade de melhor conhecer os seus clientes, de acordo com o custo e o retorno proporcionado por eles. Desta maneira, o gerenciamento da carteira de clientes desempenha papel fundamental na estratégia das empresas. A Metodologia do Gerenciamento Integrado (MGI), proposta a partir da união das metodologias do Valor do Cliente e Custo de Servir, constitui um importante sistema de informação que propicia um maior conhecimento da carteira de clientes e dos custos alocados na mesma. Permite a análise, classificação e auxilio na tomada de decisão quanto à captura, retenção dos clientes mais lucrativos e de menor custo de atendimento e descontinuidade de clientes, utilizando critérios objetivos, como margem bruta, custo de servir e potencial do cliente.

Palavras-Chave: Metodologia, Valor do Cliente, Custo de Servir, Gestão de clientes.
\end{abstract}

\begin{abstract}
The market competition racing stimulated companies to invest in the creation of new products and services as a way for differentiation. Consequently, the attendance costs had increased, rising the necessity of better knowing its customers, in accordance with the cost and profit carried by them. In this way, the customer's portfolio management plays an important role in the strategy of the companies. The Integrated Management Methodology (IMM), proposed from the union of the methodologies of Customer Value and Cost to Serve, constitutes an important information system that propitiates a better knowledge of the customer and costs, allowing for the analysis, classification and assists on decision taking related to capture, retention of most profitable and low cost customers, and discontinuity of customers, using objective criteria, as gross profit, cost to serve and customer potencial.
\end{abstract}

Key-words: Methodology, Costumer Value, Cost to Serve, Customer's Management.

\section{Introdução}

Cada vez mais as empresas têm procurado diferenciarse no mercado, através do conhecimento das necessidades de seus clientes. Este esforço demanda investimentos, como de tempo e dinheiro. Para maximizar os resultados, é fundamental apurar os reais custos de atendimento da sua carteira de clientes, bem como o valor destes.

Este artigo tem por objetivo propor uma Metodologia do Gerenciamento Integrado (MGI) e aplicá-la à carteira de clientes de uma empresa de distribuição de derivados de petróleo, que possui uma unidade de negócios de produtos químicos, denominada neste trabalho como Empresa XYZ. Tal metodologia foi concebida a partir da união de duas outras, atualmente aplicáveis no gerenciamento da carteira de clientes: Valor do Cliente e Custo de Servir (FIGUEIREDO, 2000 [2]; CHRISTOPHER, 2004 [1]). Pela simplicidade, pode ser aplicada em diversos segmentos de negócios, com a vantagem de possuir baixo custo operacional, gerando um conhecimento da carteira de clientes que normalmente as empresas não possuem.

Esse conhecimento, acoplado a um modelo de gerenciamento de portifólio de clientes, que integre na sua análise as variáveis de Margem Bruta (MB), Custo de Servir (CS) e Diferença do Valor Potencial do Cliente $(\triangle \mathrm{VPC})$, torna-se importante ferramenta para o gestor da empresa, auxiliando-o na tomada de decisão para captura, retenção e descontinuidade de clientes da empresa.

O capítulo 2 apresenta uma revisão da literatura, destacando os conceitos principais sobre valor do cliente, Custo de Servir, Gerenciamento da carteira de clientes, Segmentação de Clientes, Modelos de gerenciamento de portifólio de Clientes. O capítulo 3 apresenta a Metodologia do Gerenciamento Integrado (MGI), que foi gerada a partir da união dos conceitos de Valor do Cliente e Custo de Servir, bem como o tipo de pesquisa, método, procedimento para coleta de dados e tratamento dos dados para cada uma das metodologias. O capítulo 4 apresenta a análise dos 
dados e discussão dos resultados e no capítulo 5 Vêm as conclusões do artigo.

\section{Revisão de Literatura}

Nesta parte do trabalho são apresentados os principais campos de conhecimento que sustentam a proposição da Metodologia do Gerenciamento Integrado, cujos sob-tópicos são, nesta ordem: Valor do Cliente; Custo de Servir; Conhecimento da Carteira de Clientes - este subdividido em Vantagens do Gerenciamento da Carteira de Clientes; Decisão da captura, retenção e descontinuidade de clientes; Segmentação de Clientes e Modelos de gerenciamento de portifólio de Clientes.

\subsection{Valor do Cliente}

Ao longo do tempo, o conceito do valor da empresa passou por uma evolução, deixando de levar em conta simplesmente a soma de seus ativos (Contábil Ajustado, que contempla os bens físicos, palpáveis, que estão no ativo imobilizado), passando também a contabilizar os Fluxos de Caixa Descontados (visão financeira, menos palpável, inserindo na análise outros ativos valiosos, como, por exemplo, os clientes).

Segundo Taurion (2004) [4], o valor do cliente é o total dos valores de consumo desse cliente ao longo de sua relação com a empresa. $O$ próprio valor de mercado da empresa é influenciado pelo valor do relacionamento da empresa com seus clientes, representando garantia de suas receitas futuras. Portanto, a criação de relacionamentos estáveis e sólidos com clientes significa muito mais que apenas aumento de vendas.

Assim, a Metodologia do Valor do Cliente pode ser expressa através da equação 1 .

$$
\text { VPL Cliente }=\sum\left[\frac{(M B-C D)(1-I R)}{(1+W A C C)^{n}}\right]
$$

Sendo:

- VPL = Valor Presente Líquido

- MB = Margem Bruta

- $\mathrm{CD}=$ Custos Diretos

- IR = Impostos de Renda

- WACC $=$ Weighted Average Cost of Capital ou Custo Médio Ponderado de Capital

- $\mathrm{n}=$ tempo de análise

Dentro dessa abordagem, o valor da empresa pode ser expresso pelo somatório do valor dos clientes, abatido dos custos de manutenção dos mesmos, bem como do custo de capital empregado na empresa, como apresentado na equação 2 :

Valor Empresa $=\sum[V P L$ Clientes $-V P L C F(1-I R)-D I V]$

)

Sendo:

- $\mathrm{CF}=$ Custo Fixo
- DIV = Dívida (Capital de terceiros)

De forma simplificada no trabalho, com o horizonte da perpetuidade (nesse caso, ao trabalhar com relações de longo prazo com os clientes - fidelização), pode-se expressar o valor do cliente pela equação 3 :

$$
\text { Valor Real do Cliente }(V R C)=\frac{M B}{W A C C}
$$

O valor potencial do cliente pode ser expresso como o somatório dos vários produtos e serviços que o mesmo adquire do mercado, ilustrado na equação 4 :

$$
\text { Valor Potencial do Cliente }(V P C)=\frac{(M B 1+M B 2+\ldots M B n)}{W A C C}
$$

A participação da empresa nesses clientes (Participação no Cliente) pode ser expressa pela equação 5 , que descreve qual a fatia que se tem nesse cliente atualmente, quantificando o potencial de conversão do mesmo:

$$
\text { Participação no Cliente }(P C)=\frac{V R C}{V P C}
$$

O Valor Potencial do Cliente total da carteira de clientes da Empresa XYZ foi obtido a partir do somatório do Valor Real do Cliente dividido pela Participação Média no Cliente (PMC) de cada Segmento.

\subsection{Custo de Servir}

Segundo Tobias (2002) [5], o Custo de Servir é uma poderosa ferramenta de análise de gestão e comportamento de clientes. O objetivo principal dessa metodologia é calcular a rentabilidade por cliente de modo a identificar os clientes rentáveis e não rentáveis.

A partir dessas informações é possível identificar as causas dos clientes não rentáveis e tomar ações para que os mesmos se tornem rentáveis, alterando os demonstrativos de uma posição de prejuízo de servir para uma posição de lucro de servir.

Além desse objetivo, é possível avaliar oportunidades de melhoria em todos os elos da cadeia de suprimentos, gerenciar custos de todas as áreas da empresa e avaliar as ações tomadas.

Os métodos tradicionais dificilmente fazem uma boa análise da lucratividade por cliente, pois usualmente consideram apenas a Margem Bruta (diferença do Faturamento Líquido e dos Custos dos Produtos Vendidos) como fator de decisão (ZOLKIEWSKI, 2000) [7].

Entretanto, há uma série de outros custos que são cada vez mais significativos e que devem ser considerados para que se possa estimar a Lucratividade Real ou Lucro de Servir dos clientes. Esses custos podem ser chamados de Custos de Servir um determinado cliente. Como exemplo, podemos listar os custos de vendas e atendimento, tempo de gerenciamento da conta, custos de processo, custos logísticos, custos com manuseios, custos com financiamento, custos com transporte etc. Vale ressaltar que os custos derivados do atendimento aos 
clientes são independentes do volume de vendas para os mesmos (CHRISTOPHER, 2004, p. 104) [1].

Quando todos esses custos são perfeitamente identificados, isto é, quando se consegue calcular exatamente a lucratividade cliente por cliente, esta análise pode levar à conclusão de que há um razoável número de clientes que contribuem negativamente para a lucratividade da empresa, assim como há clientes que é preciso "proteger" dos ataques dos competidores.

Sendo assim, calcular o Custo de Servir (CS) é basicamente identificar, direcionar e alocar todos os custos necessários para o atendimento de cada cliente dessa carteira.

O Lucro de Servir (LS) está na diminuição da Margem Bruta (MB) dos clientes pelo Custo de Servir (CS) dos mesmos, como explicita a equação 6:

$$
\mathbf{L S}=\mathbf{M B}-\mathbf{C S}
$$

Essa análise revelará que alguns clientes da carteira geram contribuições negativas, e que anteriormente possuíam margem bruta positiva. Isso porque vários custos inerentes do processo de atendimento não estavam sendo alocados nos clientes em questão.

Após a apuração e alocação dos Custos de Servir por cliente, é possível representar a organização dos clientes em uma matriz, contendo quatro quadrantes, classificando os mesmos segundo a Margem Bruta e o seu Custo Servir. Essa matriz sugere algumas ações para a adoção de estratégias de gerenciamento da carteira. (CHRISTOPHER, 2004, p. 108 ) [1]. A figura 1 apresenta os quatro quadrantes da matriz MB x CS.

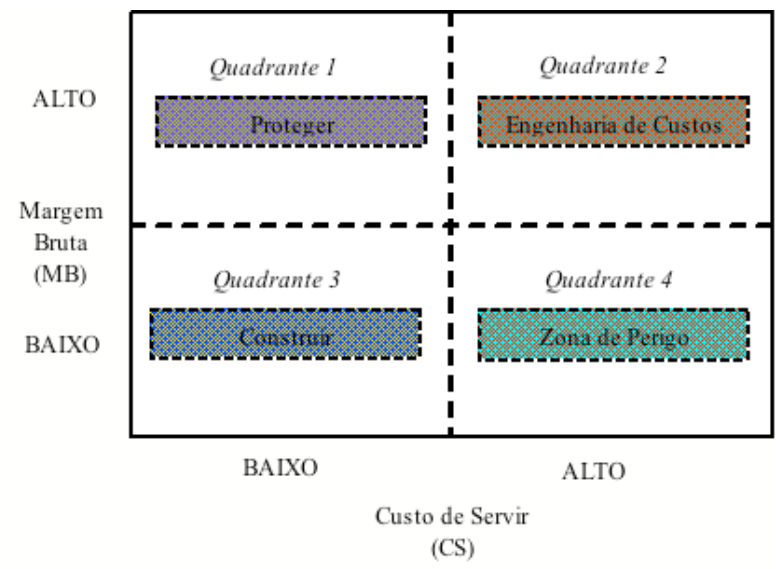

Figura 1 - Quadrantes da matriz MB x CS. Fonte: Christopher (2004) [1]

Segundo Christopher (2004) [1], é possível aplicar uma estratégia apropriada para cada tipo de quadrante da matriz, definindo determinadas ações para maximizar o resultado da carteira de clientes:

Quadrante 1: Alta $M B$ - Baixo CS

Ação: Proteger (Quadrante 1). Deve-se manter um bom relacionamento de longo prazo com esses clientes, de modo a fidelizá-los, para que não busquem outras opções de fornecimento.

Quadrante 2: Alta $M B$ - Alto CS

Ação: Engenharia de Custo (Quadrante 2). Esses clientes podem ser mais lucrativos se os custos para servi-los forem reduzidos. A diferenciação do canal de venda, como também a forma do atendimento são alternativas, fazendo-os migrar para o quadrante 1 .

Quadrante 3: Baixa MB - Baixo CS

Ação: Construir (Quadrante 3). Para este grupo, a metodologia de Valor do Cliente é importante para quantificar o potencial, checando se os mesmos podem ter sua rentabilidade aumentada, migrando para $o$ quadrante 1.

Quadrante 4: Baixa $M B$ - Alto $C S$

Ação: Zona de perigo (Quadrante 4). Esses clientes devem ser analisados cuidadosamente quanto à possibilidade de aumento de vendas e redução do custo de atendimento. Se por algum motivo estratégico não valer a pena mantê-los em carteira, deverão ser descontinuados.

\subsection{Gerenciamento da carteira de clientes}

O gerenciamento da carteira de clientes é estratégico para os negócios de uma empresa e seu estudo é subdividido em quatro subitens, intitulados: Vantagens do conhecimento da carteira de clientes; Decisão de captura, retenção e descontinuidade de clientes; Segmentação de Clientes e Modelos de Gerenciamento de portifólio de clientes.

\subsubsection{Vantagens do conhecimento da carteira de clientes}

A partir da análise da rentabilidade de clientes, que pode ser gerada aplicando-se as metodologias de Valor do Cliente e Custo de Servir, as empresas passam a enxergar uma série de oportunidades estratégicas que antes não podiam ser detectadas.

Segundo Figueiredo (2000) [2] é possível ajustar o nível de serviço oferecido quando conhecemos a rentabilidade da carteira de clientes. Os cinco pontos a serem observados estão relacionados a seguir:

- Atender melhor os clientes existentes e que são altamente rentáveis;

- Conhecer o Custo do Servir ao cliente e então cobrar pelo nível de serviço proporcionado;

- Oferecer descontos, se necessário, nas operações com clientes aos quais é possível servir com custos reduzidos;

- Negociar relações ganha-ganha entre fornecedores e compradores;

- Tentar atrair os clientes que proporcionam grandes lucros para os concorrentes.

\subsubsection{Decisão da captura, retenção e descontinuidade de clientes}

A análise da rentabilidade dos clientes revela a existência de clientes baratos e caros de servir, mas que 
são ou podem ser rentáveis porque estão dispostos a pagar pelo que recebem. De qualquer forma existem clientes que não se enquadram em nenhuma dessas categorias, bem como podem estar nos causando prejuízos. Muitas vezes não é necessário tomar decisões drásticas para abrir mão desses clientes, sendo necessário apenas um ajuste no nível de serviço, por exemplo, a não concessão de descontos, a redução ou eliminação do apoio de marketing ou técnico.

Uma questão fundamental a responder é o que fazer com a estrutura que atendia àqueles clientes. É importante notar que o fato de deixar de atender um cliente não implica, necessariamente, em redução de custos. Segundo Figueiredo (2000) [2], se esses custos não puderem ser evitados, existem algumas alternativas para utilizar a estrutura de serviço de modo eficiente:

- Melhorar o serviço e a rentabilidade dos clientes valiosos;

- Identificar formas de reduzir o custo do serviço para aqueles clientes caros de servir, mas que estão dispostos a pagar pelo que recebem;

- Aumentar o volume de vendas, sem um aumento proporcional nos custos de servir, para aqueles clientes sensíveis ao preço e que são baratos de servir.

\subsubsection{Segmentação de Clientes}

De modo a favorecer a retenção de clientes, uma alternativa prática a ser realizada é segmentar o mercado consumidor. Segundo Hijjar (2000) [3], segmentar significa dividir. A segmentação de mercado trata da subdivisão dos clientes (e potenciais clientes) em grupos distintos. Aqueles que pertencerem a um mesmo grupo deverão apresentar características similares, de forma que seja provável que apresentem um comportamento de compra similar.

Classificar os clientes em categorias facilita a adoção de estratégias apropriadas para cada segmento do mercado, uma vez que existem clientes mais valiosos que outros, e as estratégias de marketing necessárias são diferenciadas para cada caso (WEINSTEIN, 2002) [6].

Não há um método único para a segmentação de mercado. Os clientes podem ser agrupados com base nas suas características demográficas (setor de atividade a que pertence, tamanho da companhia), por suas características geográficas, ou com base nos seus comportamentos de compra (freqüência dos pedidos, volume de compras, faturamento), entre outras.

\subsubsection{Modelos de gerenciamento de portifólio de Clientes}

As teorias de gerenciamento de portifólio estavam associadas na sua origem à análise de investimentos financeiros (MARKOWITZ, 1952; SHARPE, 1963 apud ZOLKIEWSKI 2000, p. 1) [7]. Esses conceitos foram ao longo dos anos sendo adaptados para o contexto estratégico das empresas, sendo um dos mecanismos que auxiliam a tomada de decisão, bem como a alocação de recursos.
O objetivo principal dos modelos de gerenciamento de portifólio está na categorização dos relacionamentos, agrupando os clientes com determinadas características, propiciando ao gerente a possibilidade de determinar qual a melhor estratégia para otimizar o seu negócio.

Uma série de modelos de gerenciamento de portifólio sugere a análise de variáveis ligadas a fornecedores e clientes. Fiocca, Campbell e Cunningham, Shapiro et al., Krapfel et al., Olsen e Ellram e Turnbull e Zolkiewski (apud ZOLKIEWSKI, 2000 , p. 2) [7] são alguns autores que desenvolveram modelos de gerenciamento do portifólio de clientes.

Entretanto esses modelos diferem entre si pelo número e natureza das variáveis, número de etapas que o método deve ser aplicado, tipos de recomendação indicados para cada grupo de clientes categorizados etc. Outra diferenciação está na construção do modelo, o que conseqüentemente leva a uma gama de diferentes categorias criadas. Um exemplo disso são as oito categorias para classificação do cliente do modelo de Turnbull e Zolkiewski (1997), bem como quatro diferentes categorias que os modelos de Shapiro et al. (1987) e Krapfel et al. (1991) indicam (ZOLKIEWSKI, 2000) [7].

Parte das variáveis utilizadas nesses modelos são subjetivas, o que traz problemas no momento de definilas, bem como mensurá-las.

\section{Metodologia do Gerenciamento Integrado (MGI)}

A metodologia proposta foi chamada de Metodologia do Gerenciamento Integrado (MGI), unindo os conceitos de Valor do Cliente e Custo de Servir, a fim de se conhecer o Valor Potencial dos Clientes da carteira, bem como o Lucro de Servir cada cliente. A figura 2 detalha esquematicamente a Metodologia do Gerenciamento Integrado (MGI). 


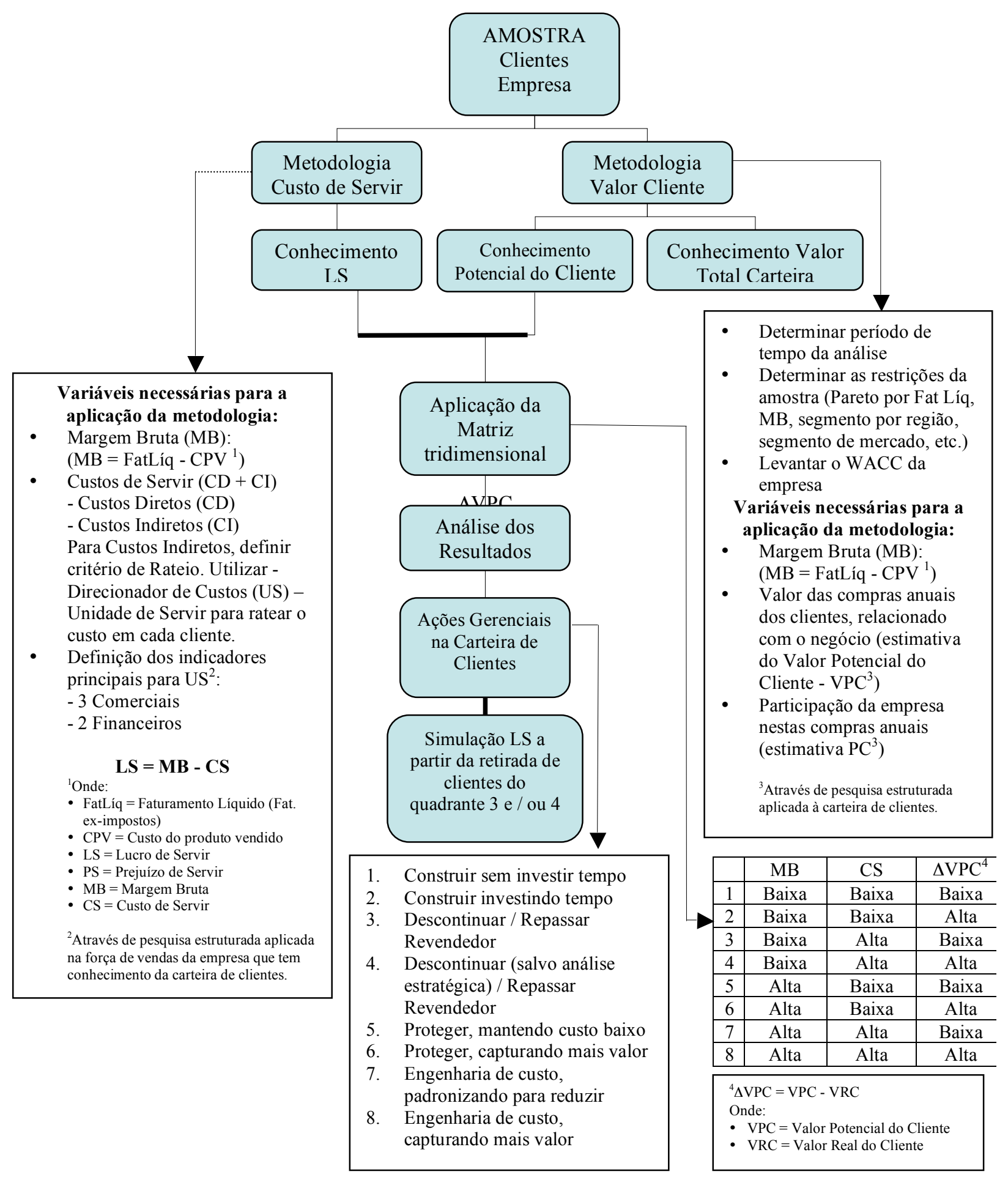

Figura 2 - Metodologia do Gerenciamento Integrado (MGI)

Após o levantamento do Valor Potencial do Cliente (a partir da metodologia do Valor do Cliente), bem como o Custo de Servir, pode-se aplicar a matriz de gerenciamento de portifólio tri-dimensional MB x CS x $\triangle \mathrm{VPC}$, com a qual se pode classificar a amostra em cada um dos seus oito quadrantes, como ilustra a figura 3.

As ações para a maximização do valor da carteira estão ligadas à estratégia de gerenciamento do portifólio de clientes a ser utilizada. Abaixo temos o detalhamento das estratégias por quadrante proposto na Metodologia MGI:

Quadrante 1: Baixa MB - Baixo CS - Baixo $\triangle$ VPC

Ação: Construir sem investir tempo. Esses clientes normalmente diluem os custos fixos da empresa. Podese pensar em reduzir ainda mais os custos de atendimento, modificando-se a forma de relacionamento (por exemplo, o atendimento passar a ser realizado através da central de telemarketing). 


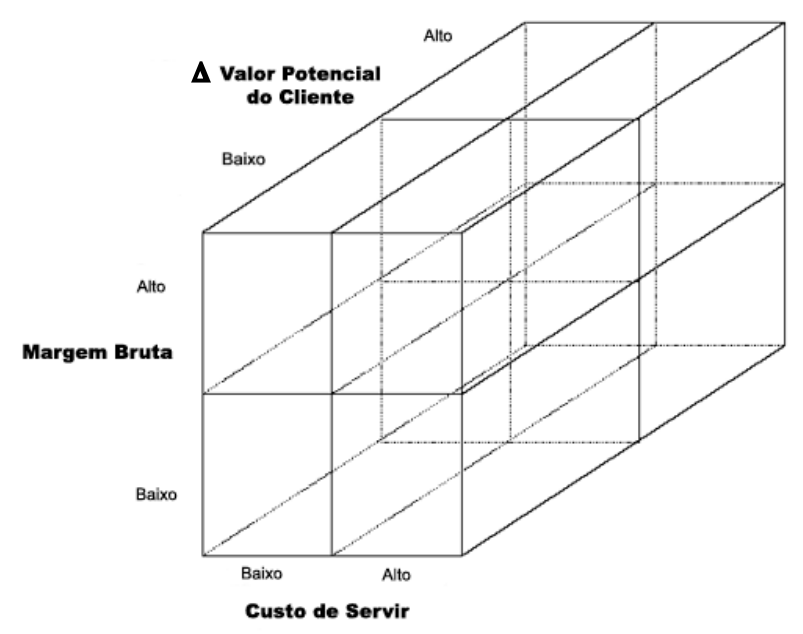

Figura 3 - Matriz tridimensional MB x CS x $\Delta$ VPC. Fonte: adaptada da matriz de Turnbull e Zokiewski (apud ZOLKIEWSKI, 2000, p. 12) [7] para a Metodologia $M G I$

\section{Quadrante 2: Baixa MB - Baixo CS - Alto $\triangle$ VPC}

Ação: Construir investindo tempo. Esses clientes são candidatos a migrarem para o quadrante 5 (Proteger, mantendo custo baixo). Se após o primeiro ciclo de análise da metodologia o cliente não tiver sido reclassificado, vale a análise de modificar a forma de relacionamento, conforme descrito no quadrante 1 .

\section{Quadrante 3: Baixa MB - Alto CS - Baixo $\triangle$ VPC}

Ação: Descontinuar / Repassar para Revendedor. Esses clientes devem ser analisados cuidadosamente, visto que pela metodologia do Valor do Cliente existe um baixo valor potencial, não podendo auxiliar na elevação da margem bruta, o que compensaria o alto custo de servir. Se os custos ligados ao atendimento não puderem ser reduzidos, a ação recomendada será descontinuá-los. Outra opção seria repassá-los para uma rede de revenda, que pode apresentar menores custos de servir.

\section{Quadrante 4: Baixa MB - Alto CS - Alto $\triangle$ VPC}

Ação: Descontinuar (salvo análise estratégica) / Repassar para Revendedor. Esses clientes devem ser analisados cuidadosamente, visto que pela metodologia do Valor do Cliente existe um alto valor potencial, que poderia auxiliar na elevação da margem bruta, compensando o seu alto custo de servir. A análise pode recomendar a migração para o quadrante 7 (Engenharia de custo, padronizando para reduzir). Se após o primeiro ciclo de análise da metodologia os clientes desse grupo não tiverem sido reclassificados, então se deve repassá-los para uma outra revenda.

Quadrante 5: Alta MB - Baixo CS - Baixo $\triangle$ VPC

Ação: Proteger, mantendo custo baixo. Esses clientes devem ser protegidos. Recomenda-se manter um bom relacionamento de longo prazo, de modo a fidelizá-los, para que não busquem outras opções de fornecimento. $\mathrm{O}$ ideal é manter os custos de atendimentos sob controle, evitando ações que possam elevar o custo de servir, pois apresentam baixo valor potencial. Se isso não for possível, padronizar, aplicando a mesma estratégia descrita para o quadrante 7 (Engenharia de custo, padronizando para reduzir).

Quadrante 6: Alta MB - Baixo CS - Alto $\triangle$ VPC

Ação: Proteger, capturando mais valor. Esses clientes devem ser protegidos, pois são muito significativos para a empresa. Deve-se manter um bom relacionamento de longo prazo, de modo a fidelizálos, para que não busquem outras opções de fornecimento. $O$ ideal é que, além de manter os custos de atendimentos, a empresa invista em ações que possam capturar mais valor, visto que apresentam alto valor potencial.Quadrante 7: Alta $M B$ - Alto $C S$ - Baixo $\triangle V P C$

Ação: Engenharia de custo, padronizando para reduzir. Esses clientes podem ser mais lucrativos se os custos para servi-los for reduzido, visto que a principal ação recomendada é a redução dos custos de servir (pois o valor potencial é baixo). A diferenciação do canal de venda, como também a forma do atendimento por ser uma saída para que se ajuste a questão dos custos, migrando-os para o quadrante 5 - Proteger, mantendo custo baixo.

Quadrante 8: Alta MB - Alto CS - Alto $\triangle$ VPC

Ação: Engenharia de custo, capturando mais valor. Esses clientes podem ser mais lucrativos se os custos de servi-los for reduzido, bem como se a captura do valor potencial puder ser realizada. A diferenciação do canal de venda, como também a forma do atendimento pode ser uma saída para a redução de custos, migrando-os para o quadrante 5 (Proteger, mantendo custo baixo) ou 7 (Engenharia de custo, padronizando para reduzir). A empresa deve também investir em ações que possam capturar mais valor, pois esses clientes apresentam alto valor potencial.

\subsection{Procedimentos para coleta de dados}

A coleta de dados para a realização desta pesquisa foi feita na unidade de negócios de produtos químicos de uma empresa distribuidora de derivados de petróleo, a $\mathrm{XYZ}$, e seus procedimentos metodológicos subdividem-se nos seguintes tópicos: Valor do Cliente e Custo de Servir

\subsubsection{Valor do Cliente}

Os dados para o cálculo do valor dos clientes foram coletados a partir da carteira de clientes ativos, isto é, todos os clientes que adquiriram produtos durante um período específico de tempo (ano base 2004). O número total de clientes encontrados com este perfil foi de 898.

Pela dificuldade em se aplicar o método na carteira como um todo, foi definido que seria utilizada a Regra de Pareto, aplicada ao faturamento líquido anual dos clientes, para seleção da amostra de pesquisa. Foram selecionados então 135 clientes que correspondiam a $80 \%$ do faturamento $(15 \%$ da amostra original).

Dividiu-se a amostra em segmentos de mercado, respeitando-se a divisão de segmentos utilizada pela 
própria área de negócios da empresa, conforme indicado na tabela 1 :

\begin{tabular}{|c|c|c|c|}
\hline Segmento & $\begin{array}{c}\text { Número } \\
\text { de } \\
\text { Clientes } \\
\text { Total } \\
\text { (NCT) }\end{array}$ & $\begin{array}{c}\text { Amostra } \\
\text { Clientes } \\
\text { Total } \\
\text { (Pareto) } \\
\text { (ACTp) }\end{array}$ & $\begin{array}{c}\text { Amostra Clientes } \\
\text { que responderam a } \\
\text { pesquisa (Pareto) } \\
\text { (ACRp) }\end{array}$ \\
\hline 1 & 22 & 0 & 0 \\
\hline 2 & 198 & 40 & 31 \\
\hline 3 & 167 & 53 & 40 \\
\hline 4 & 511 & 42 & 33 \\
\hline Total & $\mathbf{8 9 8}$ & $\mathbf{1 3 5}$ & $\mathbf{1 0 4}$ \\
\hline
\end{tabular}

Tabela 1 - Segmentos analisados X Número de

Clientes. Fonte: Dados do relatório de vendas da empresa

Não se aplicou a metodologia no Segmento 1 por indicação da Empresa XYZ, visto que a mesma já dispunha de informações sobre o Valor Potencial deste grupo de Clientes (VPC), bem como da Participação no Cliente (PC).

Após a definição da amostra, foram realizados os seguintes passos:

1. Levantamento dos principais dados cadastrais desses clientes para criação e implementação de um banco de dados com as informações levantadas e as posteriormente obtidas (como: nome do cliente; endereço; CNPJ; telefones e correio eletrônico dos responsáveis pela aquisição de produtos químicos; produtos atualmente adquiridos; vendedores responsáveis pelo cliente etc.);

2. Realização de contato telefônico com todos os clientes selecionados na amostra, de modo a atualizar o cadastro e informá-los sobre a execução de uma pesquisa, a ser feita, por meio de questionário, enviada por correio eletrônico (quest mail);

3. As perguntas que compuseram o questionário de pesquisa estão listadas a seguir:

- Pergunta 1: Dentre os grupos de produtos abaixo, informe o valor total de compras da empresa (clientes da Empresa XYZ) por ano - Estimativa VPC;

- Pergunta 2: Dentre os grupos de produtos abaixo, informe o percentual da Empresa XYZ dentro do valor de compras total da empresa (clientes da Empresa XYZ) por ano - Estimativa PC;

- Pergunta 3: Principais produtos adquiridos; e

- Pergunta 4: Serviços demandados ou que atualmente já adquire.

4. Envio da pesquisa por correio eletrônico pelo serviço chamado de questionário eletrônico (quest mail) para todos os responsáveis cadastrados;

5. Após encerramento do prazo da pesquisa (30 dias úteis), os dados recebidos foram coletados $\mathrm{e}$ armazenados no banco de dados. Entretanto, como o volume das respostas foi considerado insuficiente (menos de $35 \%$ da amostra original), realizou-se novo contato telefônico, aplicando-se as perguntas indicadas no item 3, com os clientes que não haviam respondido ou que responderam de forma inadequada;
6. Compilação da massa de dados obtida ao final dos contatos e cálculo dos valores real e potencial dos clientes em questão.

$\mathrm{O}$ número de respondentes total ao final da pesquisa foi de 104 empresas, perfazendo um faturamento líquido acumulado de 75,3\%.

\subsubsection{Custo de Servir}

A amostra para o cálculo do custo de servir foi a mesma utilizada na tabela 1 .

Posteriormente, foram realizados os passos descritos abaixo:

1. Levantamento da lista de todos os clientes da carteira da Empresa XYZ (898 clientes ativos), contendo informações sobre o Segmento, Nome do Vendedor, Nome do Cliente, Código do Cliente na empresa, Volume, Zona de Vendas (agrupamento de clientes, gerida por um Vendedor), Faturamento e Margem Bruta total dos produtos adquiridos.

2. Coleta dos Custos Diretos e definição dos critérios de rateio dos Custos Indiretos:

- Custos Diretos: Pessoal / Frete Fornecimento / Despesas Gerais etc. alocados até a Zona de Vendas.

- Custos Indiretos: Overhead - gerentes de vendas do segmento, estrutura de marketing e planejamento, gerência executiva, estrutura operacional (depósitos, bases e terminais) e da empresa como um todo.

3. Formatação de uma pesquisa aplicada a cada membro da força de venda (Vendedores), contendo 5 perguntas, para definição da alocação de custos por cliente - criação do direcionador de custos, chamado Unidade de Servir (US).

Os campos que constaram no questionário foram: Vendedor, Zona de Vendas, Nome do Cliente, Código do Cliente, Pergunta 1, Pergunta 2, Pergunta 3, Pergunta 4, Pergunta 5 e Observações.

Os Critérios para elaboração do direcionador de custo - Unidade de Servir (US) são listados abaixo:

- Pergunta 1: Grau de "envolvimento" da Força de vendas no atendimento ao cliente ( 1 a 4 pontos);

- Pergunta 2: Grau de "envolvimento" do Gerente de Segmento no atendimento ao cliente (1 a 4 pontos);

- Pergunta 3: Grau de "envolvimento" da Central de apoio a vendas (colocação dos pedidos, acompanhamento logístico, acompanhamento do pagamento, registro das informações de vendas etc.) no atendimento ao cliente (1 a 4 pontos);

- Pergunta 4: Dificuldade de obter / manter atualizadas informações cadastrais (0 a 4 pontos);

- Pergunta 5: Dificuldade de obter os recebíveis (0 a 4 pontos).

Entende-se "envolvimento" o tempo dispensado por cada interlocutor junto a cada cliente. A escala de pontuação foi dividida da seguinte forma: Nenhum $=0$ pt; Muito Baixo $=1 \mathrm{pt}$; Baixo $=2 \mathrm{pt}$; Médio $=3 \mathrm{pt}$; Alto $=4 \mathrm{pt}$

4. Envio da pesquisa por correio eletrônico para todos os responsáveis pelo gerenciamento da carteira de clientes (vendedores); 
5. Armazenamento das informações coletadas em banco de dados.

A fim de aplicar a metodologia proposta, foi selecionada a mesma amostra de clientes (104 clientes) utilizada na metodologia do Valor do Cliente.

Os cálculos realizados utilizaram as expressões da Metodologia do Custo de Servir, levando-se em conta os Custos Diretos e Indiretos. Os Custos Diretos anuais puderam ser levantados até o nível de Zona de Vendas. Os Custos Diretos que foram levados em consideração encontram-se descritos no quadro 1.

Custeio direto da

Zona de Vendas

\section{Frete \\ Pessoal \\ Outras \\ Despesas Serviços Contratados \\ Despesas Gerais \\ Publicidade e Cultura \\ Material \\ Depreciação / \\ Amortização \\ Despesas Tributárias}

Quadro 1 - Custeio Direto da Zona de Vendas

Os Custos Indiretos anuais foram rateados até o nível da Zona de Vendas através do critério de Custeio por absorção, utilizando o direcionador de custos R\$/Volume de vendas. No caso da Empresa XYZ, o volume de vendas foi expresso $\mathrm{em}^{3}$.

Os Custos Indiretos que foram levados em consideração encontram-se descritos no quadro 2 .

Após a alocação de todos os custos (diretos e indiretos) até o nível da Zona de Vendas, foi aplicado o critério de Custeio por Atividade, a fim de se ratear o custo total da Zona de Vendas para cada um dos clientes que compõem a mesma, achando-se, assim, o Custo de Servir desse determinado cliente.

As atividades que compõem o direcionador de custo US (Unidade de Servir) podem ser expressas em função do tempo dedicado de cada agente (força de vendas, gerente do segmento, central de apoio a vendas), bem como do nível de problemas ligados à disponibilidade de garantias e inadimplência, para atendimento de determinado cliente.

O direcionador de custo Unidade de Servir (US) é expresso pelo somatório das pontuações obtidas para cada cliente, segundo os critérios estabelecidos, variando de 3 a 20 pontos (quanto maior a pontuação, maior o custo de servir a ser direcionado para o cliente).

Logo, o Custo anual de Servir do Cliente pode ser expresso pela equação 7 .

$$
C S=\left[\frac{\text { número de US do cliente }}{\text { número de US dos } n \text { clientes }}\right] C T
$$

\begin{tabular}{|c|c|}
\hline $\begin{array}{c}\text { Overhead } \\
\text { Segmento } 1\end{array}$ & \multirow{4}{*}{$\begin{array}{l}\text { Todos os custos não alocados } \\
\text { diretamente na zona de vendas } \\
\text { (custos gerenciais, estrutura de apoio } \\
\text { etc.). }\end{array}$} \\
\hline $\begin{array}{c}\text { Overhead } \\
\text { Segmento } 2\end{array}$ & \\
\hline $\begin{array}{c}\text { Overhead } \\
\text { Segmento } 3\end{array}$ & \\
\hline $\begin{array}{c}\text { Overhead } \\
\text { Segmento } 4\end{array}$ & \\
\hline $\begin{array}{l}\text { Overhead } \\
\text { Unidade de } \\
\text { Negócios }+ \\
\text { Marketing + } \\
\text { Planejamento }\end{array}$ & $\begin{array}{l}\text { Todos os custos não alocados } \\
\text { diretamente na zona de vendas } \\
\text { (custos gerenciais, estrutura de apoio } \\
\text { etc.). }\end{array}$ \\
\hline $\begin{array}{l}\text { Overhead } \\
\text { Depósitos }\end{array}$ & $\begin{array}{l}\text { Todos os custos não alocados } \\
\text { diretamente na zona de vendas } \\
\text { (custos operacionais, estrutura de } \\
\text { apoio etc.). Apenas o Segmento } 1 \\
\text { utiliza esta estrutura operacional. }\end{array}$ \\
\hline $\begin{array}{c}\text { Overhead } \\
\text { Operacional }\end{array}$ & $\begin{array}{l}\text { Todos os custos não alocados } \\
\text { diretamente na zona de vendas } \\
\text { (custos operacionais, estrutura de } \\
\text { apoio etc.). Apenas os Segmentos } 2 \text {, } \\
3 \text { e } 4 \text { utilizam esta estrutura } \\
\text { operacional. }\end{array}$ \\
\hline $\begin{array}{c}\text { Overhead } \\
\text { Companhia }\end{array}$ & $\begin{array}{l}\text { Todos os custos não alocados } \\
\text { diretamente na zona de vendas } \\
\text { (custos gerenciais, estrutura de apoio } \\
\text { etc.). }\end{array}$ \\
\hline
\end{tabular}

Quadro 2 - Custeio Indireto da Zona de Vendas

Sendo:

- $\mathrm{CS}=$ Custo de Servir do Cliente

- Número de US do cliente = pontuação do cliente

- Número de US dos $\mathrm{n}$ clientes = somatório da pontuação de todos os clientes de uma determinada Zona de Vendas

- $\mathrm{n}=$ número de clientes dentro de uma determinada Zona de Vendas

- $\mathrm{CT}=$ Custeio Total (Custos Diretos + Indiretos) alocados na Zona de Vendas

O Lucro ou Prejuízo de Servir é calculado apenas subtraindo-se a Margem Bruta do Cliente, pelo Custo de Servir do mesmo.

\section{$4 \quad$ Resultados}

Os resultados encontrados para a matriz tridimensional MB x CS x $\triangle$ VPC para toda a amostra de pesquisa, após a aplicação da Metodologia do Gerenciamento Integrado (MGI), podem ser visualizados na tabela 2 , onde:

- Segmento = identificação do segmento da Empresa $\mathrm{XYZ}$

- Cliente = indicação numérica do cliente

- $\mathrm{MB}=$ Margem Bruta

- $\mathrm{CS}=$ Custo de Servir

- $\mathrm{LS}=$ Lucro de Servir

- $\triangle \mathrm{VPC}=$ Diferença Valor Potencial do Cliente 
- $\mathrm{PC}=$ Participação no cliente

- Quadrante = Região onde cada cliente foi alocado em função dos dados de MB, CS e $\triangle \mathrm{VPC}$ levantados pela metodologia MGI

- Quadrante = Simulação levando-se em consideração que todo $\triangle \mathrm{VPC}$ fosse capturado pela Empresa XYZ

\subsection{Comentários sobre a classificação}

A metodologia MGI pode ser aplicada em qualquer carteira de clientes desde que os dados de lucratividade (Margem Bruta), custo de capital médio ponderado (WACC), custos diretos e indiretos possam ser contabilizados e explicitados pela empresa.

Foi feita uma simulação (coluna QUAD ${ }^{\mathrm{TE}}$ da tabela 2) checando-se o posicionamento dos clientes da amostra de pesquisa se o $\triangle \mathrm{VPC}$ fosse todo capturado pela empresa XYZ (assumindo que os custos de servir não aumentariam significativamente com esta ação). Como ação estratégica a empresa XYZ deveria trabalhar prioritariamente os clientes 9 e 10 do segmento \#3, bem como o cliente 3 do segmento \#4, pois passariam para o quadrante 6 (Alta MB, Baixo CS e Alto $\triangle \mathrm{VPC}$ - proteger capturando mais valor).

A aplicação da metodologia MGI fornece apenas um quadro pontual de como está organizada a carteira de clientes de uma empresa, classificando cada cliente nos quadrantes indicados na metodologia. A análise de tendências somente poderá ser realizada se a metodologia for aplicada em mais de um período de tempo.

Outro ponto que deve ser observado é a alocação dos custos para cálculo do Custo de Servir. Em função da definição dos critérios de rateio para alocação dos Custos Indiretos (ABC, Custeio por absorção etc.), pode haver variações na classificação dos clientes nos quadrantes, principalmente daqueles que possuem Lucro de Servir próximos ao Custo de Servir, levando a uma possível distorção na análise.

Quanto ao levantamento do Valor do Cliente, como o mesmo é baseado em pesquisa junto aos clientes da empresa, se a qualidade das respostas, bem como a escolha do grupo de respondentes não for ajustada, têm-se distorções no cálculo do Valor do Cliente, impactando a qualidade dos resultados coletados.

Seria muito interessante a possibilidade de aplicação da Metodologia do Valor do Cliente para toda a carteira da Empresa XYZ. Isso possibilitaria a análise e aplicação da Metodologia do Gerenciamento Integrado (MGI) para a carteira como um todo, possibilitando enriquecer o conhecimento dos clientes que apresentaram Prejuízo de Servir, auxiliando de forma objetiva na decisão acerca das ações a serem tomadas (manutenção ou descontinuidade).

Mesmo com os pontos observados é notório que ao inserir-se a variável $\triangle \mathrm{VPC}$ na análise do cliente, conforme proposto pela Metodologia MGI e testado na amostra de pesquisa, foi possível ter um conhecimento objetivo de quais clientes têm ou não potencial para migrar de um quadrante para outro, reduzindo assim o tempo de análise e decisão.

\section{Conclusões}

As Metodologias de Gerenciamento de Carteira de Clientes desempenham papel fundamental na estratégia gerencial das empresas, sendo possível extrair informações relevantes tanto dos dados internos da empresa, como junto aos próprios clientes.

A maximização do valor da carteira de clientes pode ser conseguida quando se conhece o valor do cliente e seus custos de servir. Com estas duas informações pode-se classificá-lo, aplicando-se, posteriormente, uma estratégia de gerenciamento de portifólio, auxiliando o gestor da empresa quanto à manutenção ou descontinuidade de determinados clientes.

As metodologias do Valor do Cliente e Custo de Servir são eficazes para a classificação de uma carteira de clientes, maximizar o seu valor, bem como na tomada de decisão. Quando as mesmas são unidas, como proposto neste artigo, através da Metodologia do Gerenciamento Integrado (MGI), pode-se ampliar a visão da carteira, auxiliando o gestor a tomar decisões pautadas em fatos objetivos, que podem ser obtidos de forma simples, por meio dos dados de vendas e custos que normalmente as empresas monitoram.

O conhecimento da carteira de clientes auxilia a empresa a direcionar os seus esforços na captura desse valor, bem como indica as análises a serem efetuadas, indicadas a partir da classificação dos clientes nos oito quadrantes propostos na metodologia MGI.

Normalmente, uma carteira de clientes é heterogênea. Caso não se consiga ter um critério de agrupamento, encontrando um ponto comum para tratá-los, bem como se ter ações estratégicas definidas em cada um dos quadrantes, torna-se difícil a aplicação de qualquer metodologia de gerenciamento, o que gera, muitas vezes, perdas de recursos para as empresas.

Outro ponto a ser ressaltado refere-se ao baixo custo operacional para aplicação do método. Basicamente seria necessária uma equipe da empresa que conhecesse de que modo as vendas e os custos são apurados, bem como a alocação de um profissional para executar a pesquisa junto aos clientes (para levantamento do VPC) e junto à força de vendas (para levantamento da US). 


\begin{tabular}{|c|c|c|c|c|c|c|c|c|}
\hline $\mathrm{SEG}^{\mathrm{TO}}$ & CLIENTE & MB & $\mathrm{CS}$ & LS & $\Delta \mathrm{VPC}$ & $\mathrm{PC}$ & QUAD $^{\text {TE }}$ & $\mathrm{QUAD}^{\mathrm{TE}}$ \\
\hline$\# 2$ & \begin{tabular}{|l|}
1 \\
\end{tabular} & 851.270 & 68.590 & 782.680 & 836.137 & \begin{tabular}{|l|}
$86 \%$ \\
\end{tabular} & 5 & 5 \\
\hline$\# 2$ & 2 & 231.467 & 42.030 & 189.436 & 5.414 .428 & $20 \%$ & 1 & 5 \\
\hline$\# 2$ & 3 & 658.934 & 62.350 & 596.585 & 3.853 .418 & $50 \%$ & 1 & 5 \\
\hline$\# 2$ & 4 & 417.560 & 63.046 & 354.515 & 629.565 & $80 \%$ & 1 & 5 \\
\hline$\# 2$ & 5 & 90.626 & 54.640 & 35.986 & 353.318 & $60 \%$ & 1 & 1 \\
\hline$\# 2$ & 6 & 60.934 & 54.640 & 6.294 & 237.558 & $60 \%$ & 1 & 1 \\
\hline$\# 2$ & 7 & 598.820 & 31.340 & 567.480 & 184.309 & $95 \%$ & 1 & 5 \\
\hline$\# 2$ & 8 & 106.289 & 40.153 & 66.135 & 160.254 & $80 \%$ & 1 & 1 \\
\hline$\# 2$ & 9 & 32.322 & 54.640 & $(22.318)$ & 126.012 & $60 \%$ & 1 & 1 \\
\hline$\# 2$ & 10 & 390.990 & 80.164 & 310.826 & 120.342 & $95 \%$ & 1 & 1 \\
\hline$\# 2$ & 11 & 292.043 & 53.443 & 238.600 & 89.887 & $95 \%$ & 1 & 1 \\
\hline$\# 2$ & 12 & 286.552 & 58.843 & 227.709 & 88.197 & $95 \%$ & 1 & 1 \\
\hline$\# 2$ & 13 & 272.020 & 23.874 & 248.146 & 83.724 & $95 \%$ & 1 & 1 \\
\hline$\# 2$ & 14 & 264.154 & 31.340 & 232.814 & 81.303 & 95\% & 1 & 1 \\
\hline$\# 2$ & 15 & 239.957 & 97.978 & 141.979 & 73.856 & $95 \%$ & 1 & 1 \\
\hline$\# 2$ & 16 & 234.998 & 53.443 & 181.556 & 72.329 & $95 \%$ & 1 & 1 \\
\hline$\# 2$ & 17 & 229.040 & 60.133 & 168.908 & 70.496 & $95 \%$ & 1 & 1 \\
\hline$\# 2$ & 18 & 165.737 & 62.350 & 103.387 & 51.012 & $95 \%$ & 1 & 1 \\
\hline$\# 2$ & 19 & 158.635 & 51.442 & 107.193 & 48.826 & $95 \%$ & 1 & 1 \\
\hline$\# 2$ & 20 & 151.876 & 60.133 & 91.743 & 46.745 & $95 \%$ & 1 & 1 \\
\hline$\# 2$ & 21 & 123.624 & 60.133 & 63.491 & 38.050 & $95 \%$ & 1 & 1 \\
\hline$\# 2$ & 22 & 122.189 & 37.285 & 84.903 & 37.608 & \begin{tabular}{|l|}
$95 \%$ \\
\end{tabular} & 1 & 1 \\
\hline$\# 2$ & 23 & 116.466 & 26.117 & 90.349 & 35.847 & $95 \%$ & 1 & 1 \\
\hline$\# 2$ & 24 & 107.333 & 26.117 & 81.216 & 33.036 & $95 \%$ & 1 & 1 \\
\hline$\# 2$ & 25 & 95.233 & 37.285 & 57.948 & 29.312 & $95 \%$ & 1 & 1 \\
\hline$\# 2$ & 26 & 89.209 & 51.442 & 37.767 & 27.457 & $95 \%$ & 1 & 1 \\
\hline$\# 2$ & 27 & 89.209 & 26.117 & 63.093 & 27.457 & $95 \%$ & 1 & 1 \\
\hline$\# 2$ & 28 & 76.457 & 28.540 & 47.916 & 23.532 & $95 \%$ & 1 & 1 \\
\hline$\# 2$ & 29 & 53.300 & 26.117 & 27.183 & 16.405 & $95 \%$ & 1 & 1 \\
\hline$\# 2$ & 30 & 47.699 & 58.843 & $(11.144)$ & 14.681 & $95 \%$ & 1 & 1 \\
\hline \#2 & 31 & 11.034 & 37.285 & $(26.251)$ & 3.396 & $95 \%$ & 1 & 1 \\
\hline$\# 3$ & 1 & 1.517 .305 & 178.867 & 1.338 .438 & 467.007 & $95 \%$ & 7 & 7 \\
\hline$\# 3$ & 2 & 1.420 .554 & 51.973 & 1.368 .581 & 19.383 .778 & $30 \%$ & 6 & 6 \\
\hline$\# 3$ & 3 & 781.548 & 107.596 & 673.952 & 1.958 .767 & $70 \%$ & 5 & 5 \\
\hline$\# 3$ & 4 & 850.210 & 48.261 & 801.949 & 552.443 & $90 \%$ & 5 & 5 \\
\hline$\# 3$ & 5 & 639.002 & 131.507 & 507.496 & 11.210 .568 & $25 \%$ & 4 & 8 \\
\hline$\# 3$ & 6 & 308.897 & 216.471 & 92.426 & 1.204 .276 & $60 \%$ & 3 & 7 \\
\hline$\# 3$ & 7 & 174.361 & 136.781 & 37.580 & 1.019 .656 & $50 \%$ & 3 & 7 \\
\hline$\# 3$ & 8 & 304.888 & 168.346 & 136.542 & 445.742 & $80 \%$ & 3 & 3 \\
\hline$\# 3$ & 9 & 110.128 & 29.699 & 80.428 & 12.236 .400 & $5 \%$ & 2 & 6 \\
\hline$\# 3$ & 10 & 304.835 & 42.789 & 262.046 & 10.101 .738 & $15 \%$ & 2 & 6 \\
\hline$\# 3$ & 11 & 233.873 & 107.596 & 126.276 & 5.470 .709 & $20 \%$ & 1 & 5 \\
\hline$\# 3$ & 12 & 388.200 & 64.941 & 323.259 & 5.293 .193 & $30 \%$ & 1 & 5 \\
\hline$\# 3$ & 13 & 379.997 & 39.498 & 340.499 & 5.185 .142 & $30 \%$ & 1 & 5 \\
\hline$\# 3$ & 14 & 200.606 & 64.941 & 135.665 & 4.692 .547 & $20 \%$ & 1 & 5 \\
\hline$\# 3$ & 15 & 311.233 & 29.842 & 281.391 & 2.730 .116 & $40 \%$ & 1 & 5 \\
\hline$\# 3$ & 16 & 139.667 & 47.821 & 91.846 & 2.434 .369 & $25 \%$ & 1 & 5 \\
\hline$\# 3$ & 17 & 76.901 & 42.789 & 34.112 & 2.235 .148 & $17 \%$ & 1 & 5 \\
\hline$\# 3$ & 18 & 309.262 & 94.694 & 214.567 & 1.812 .200 & $50 \%$ & 1 & 5 \\
\hline$\# 3$ & 19 & 89.018 & 48.203 & 40.815 & 1.561 .726 & $25 \%$ & 1 & 5 \\
\hline$\# 3$ & 20 & 251.204 & 44.549 & 206.656 & 1.469 .032 & $50 \%$ & 1 & 5 \\
\hline$\# 3$ & 21 & 678.286 & 94.694 & 583.591 & 1.322 .194 & $75 \%$ & 1 & 5 \\
\hline$\# 3$ & 22 & 225.990 & 71.731 & 154.259 & 1.321 .579 & $50 \%$ & 1 & 5 \\
\hline$\# 3$ & 23 & 398.104 & 64.271 & 333.833 & 997.753 & $70 \%$ & 1 & 5 \\
\hline$\# 3$ & 24 & 184.171 & 95.641 & 88.530 & 881.202 & $55 \%$ & 1 & 5 \\
\hline
\end{tabular}




\begin{tabular}{|c|c|c|c|c|c|c|c|c|}
\hline $\mathrm{SEG}^{\mathrm{TO}}$ & CLIENTE & MB & $\mathrm{CS}$ & LS & $\triangle \mathrm{VPC}$ & $\mathrm{PC}$ & QUAD $^{\text {TE }}$ & QUAD $^{\text {TE' }^{\prime}}$ \\
\hline$\# 3$ & 25 & 186.850 & 95.641 & 91.208 & 728.458 & $60 \%$ & 1 & 5 \\
\hline$\# 3$ & 26 & 80.621 & 8.562 & 72.059 & 707.200 & $40 \%$ & 1 & 5 \\
\hline$\# 3$ & 27 & 335.125 & 37.124 & 298.001 & 660.851 & $75 \%$ & 1 & 5 \\
\hline$\# 3$ & 28 & 252.610 & 64.941 & 187.668 & 633.107 & $70 \%$ & 1 & 5 \\
\hline$\# 3$ & 29 & 96.054 & 44.549 & 51.505 & 561.719 & $50 \%$ & 1 & 1 \\
\hline$\# 3$ & 30 & 292.315 & 83.686 & 208.629 & 427.361 & $80 \%$ & 1 & 1 \\
\hline$\# 3$ & 31 & 8.119 & 20.644 & $(12.525)$ & 269.057 & $15 \%$ & 1 & 1 \\
\hline$\# 3$ & 32 & 37.941 & 35.865 & 2.076 & 221.877 & $50 \%$ & 1 & 1 \\
\hline$\# 3$ & 33 & 640.342 & 39.498 & 600.844 & 197.089 & $95 \%$ & 1 & 5 \\
\hline$\# 3$ & 34 & 208.504 & 26.332 & 182.172 & 135.480 & $90 \%$ & 1 & 1 \\
\hline$\# 3$ & 35 & 24.207 & 95.641 & $(71.434)$ & 115.823 & $55 \%$ & 1 & 1 \\
\hline$\# 3$ & 36 & 301.034 & 9.634 & 291.400 & 92.654 & $95 \%$ & 1 & 1 \\
\hline$\# 3$ & 37 & 121.096 & 71.731 & 49.365 & 78.685 & $90 \%$ & 1 & 1 \\
\hline$\# 3$ & 38 & 2.479 & 42.789 & $(40.310)$ & 72.059 & $17 \%$ & 1 & 1 \\
\hline$\# 3$ & 39 & 214.693 & 83.686 & 131.007 & 66.080 & $95 \%$ & 1 & 1 \\
\hline$\# 3$ & 40 & 98.200 & 48.203 & 49.996 & 63.807 & $90 \%$ & 1 & 1 \\
\hline$\# 4$ & 1 & 1.259 .922 & 57.104 & 1.202 .818 & 3.157 .699 & $70 \%$ & 5 & 5 \\
\hline$\# 4$ & 2 & 842.977 & 3.235 & 839.742 & 259.457 & $95 \%$ & 5 & 5 \\
\hline$\# 4$ & 3 & 126.319 & 33.326 & 92.993 & 14.035 .467 & $5 \%$ & 2 & 6 \\
\hline$\# 4$ & 4 & 435.294 & 3.235 & 432.059 & 7.636 .737 & $25 \%$ & 1 & 5 \\
\hline$\# 4$ & 5 & 679.951 & 86.647 & 593.304 & 7.384 .599 & $35 \%$ & 1 & 5 \\
\hline$\# 4$ & 6 & 143.892 & 54.227 & 89.665 & 6.229 .151 & $12 \%$ & 1 & 5 \\
\hline$\# 4$ & 7 & 130.980 & 9.461 & 121.519 & 5.670 .185 & $12 \%$ & 1 & 5 \\
\hline$\# 4$ & 8 & 241.135 & 19.908 & 221.227 & 5.640 .589 & $20 \%$ & 1 & 5 \\
\hline \#4 & 9 & 312.893 & 14.050 & 298.843 & 3.398 .167 & $35 \%$ & 1 & 5 \\
\hline \#4 & 10 & 299.434 & 14.050 & 285.384 & 3.251 .994 & $35 \%$ & 1 & 5 \\
\hline$\# 4$ & 11 & 207.932 & 46.480 & 161.452 & 2.699 .807 & $31 \%$ & 1 & 5 \\
\hline$\# 4$ & 12 & 134.024 & 15.455 & 118.570 & 2.351 .305 & $25 \%$ & 1 & 5 \\
\hline \#4 & 13 & 590.432 & 59.986 & 530.446 & 2.319 .515 & $60 \%$ & 1 & 5 \\
\hline$\# 4$ & 14 & 226.276 & 59.986 & 166.289 & 1.984 .874 & $40 \%$ & 1 & 5 \\
\hline$\# 4$ & 15 & 317.382 & 66.652 & 250.730 & 1.237 .357 & $60 \%$ & 1 & 5 \\
\hline$\# 4$ & 16 & 699.840 & 38.733 & 661.107 & 1.023 .158 & $80 \%$ & 1 & 5 \\
\hline$\# 4$ & 17 & 146.858 & 39.991 & 106.867 & 858.821 & $50 \%$ & 1 & 5 \\
\hline$\# 4$ & 18 & 19.218 & 9.461 & 9.757 & 831.956 & $12 \%$ & 1 & 5 \\
\hline$\# 4$ & 19 & 190.013 & 53.321 & 136.691 & 476.223 & $70 \%$ & 1 & 1 \\
\hline$\# 4$ & 20 & 559.822 & 79.982 & 479.840 & 472.254 & $87 \%$ & 1 & 1 \\
\hline$\# 4$ & 21 & 138.917 & 14.050 & 124.867 & 348.162 & $70 \%$ & 1 & 1 \\
\hline \#4 & 22 & 219.552 & 71.670 & 147.882 & 320.982 & $80 \%$ & 1 & 1 \\
\hline$\# 4$ & 23 & 353.666 & 99.977 & 253.689 & 283.182 & $88 \%$ & 1 & 1 \\
\hline$\# 4$ & 24 & 188.621 & 42.828 & 145.793 & 196.238 & $85 \%$ & 1 & 1 \\
\hline$\# 4$ & 25 & 72.001 & 46.656 & 25.345 & 180.454 & $70 \%$ & 1 & 1 \\
\hline$\# 4$ & 26 & 438.608 & 66.652 & 371.957 & 134.998 & $95 \%$ & 1 & 1 \\
\hline$\# 4$ & 27 & 388.491 & 73.317 & 315.174 & 119.572 & $95 \%$ & 1 & 1 \\
\hline$\# 4$ & 28 & 346.801 & 3.235 & 343.566 & 106.741 & $95 \%$ & 1 & 1 \\
\hline$\# 4$ & 29 & 188.780 & 47.780 & 141.000 & 58.104 & $95 \%$ & 1 & 1 \\
\hline$\# 4$ & 30 & 186.413 & 6.804 & 179.609 & 57.375 & $95 \%$ & 1 & 1 \\
\hline$\# 4$ & 31 & 169.319 & 42.828 & 126.491 & 52.114 & $95 \%$ & 1 & 1 \\
\hline \#4 & 32 & 93.968 & 3.235 & 90.734 & 28.922 & $95 \%$ & 1 & 1 \\
\hline$\# 4$ & 33 & 6.647 & 99.977 & $(93.331)$ & 5.322 & $88 \%$ & 1 & 1 \\
\hline
\end{tabular}

Tabela 2: Segmentos \#2, \#3 e \#4 - Matriz tri-dimensional MB x CS x AVPC

As principais variáveis a serem mapeadas para que se obtenha o valor do cliente e custo de servir, estão resumidas na metodologia do gerenciamento integrado (MGI), apresentada na figura 2. Para o valor do cliente, a variável principal é o valor potencial do cliente (VPC), que pode ser estimado a partir do valor real do cliente (margem bruta), bem como, por meio do percentual de participação que atualmente a empresa possui no mesmo. No custo de servir (CS), as variáveis 
principais estão ligadas à alocação dos custos diretos e indiretos e à margem bruta da carteira de clientes.

\section{Referências}

[1] CHRISTOPHER, Martin. Logistics e Supply Chain Management: creating value-adding networks. Prentice Hall, 3rd Edition, p.81-114, 2004.

[2] Figueiredo, K. Rentabilidade de Clientes e Nível de Serviço. In FLEURY, P.F.; WANKE, P.; FIGUEIREDO, K.F. (orgs). Logística Empresarial: a perspectiva brasileira. São Paulo: Atlas, p. 65-76, 2000.

[3] HIJJAR, Maria Fernanda. Segmentação de Mercado para Diferenciação dos Serviços Logísticos. 2000. Centro de Estudos em Logística - COPPEAD - UFRJ. Disponível em: $<$ http://www.cel.coppead.ufrj.br/fs-busca.htm?frpublic.htm>. Acesso em: 18 Nov. 2005.

[4] TAURION, Cezar (2004) A empresa centrada no cliente. IBM Business Center. Disponível em: $<$ http://www.ibm.com.br/businesscenter/articles_e tips/oa3.phtml>. Acesso em: 05 Mar. 2005.

[5] TOBIAS, A., Custo de Servir: Como calcular a rentabilidade por cliente e determinar o nível adequado de serviços, UP-TO-DATE, $\mathrm{N}^{0} 252$, 2002, p.1-19.

[6] WEINSTEIN, A., Customer-specific strategies Customer retention: $A$ usage segmentation and customer value approach, Journal of Targeting; London, Vol. 10, $\mathrm{N}^{\mathrm{o}} 3$, 2002, p. 259-268.

[7] ZOLKIEWSKI, J.; TURNBULL, P. (2000), Relationship Portfolios - Past, Present and Future, 16th International IMP Conference, Universities of Bath and Bristol, U.K., p. 1-12. 